3. Erdemir U, Sancakli H S, Yaman B C, Ozel S, Yucel T, Yildiz E. Clinical comparison of a flowable composite and fissure sealant: a 24-month split-mouth, randomized, and controlled study. J Dent 2014; 42: 149-157.

4. Kucukyilmaz E, Savas S. Evaluation of Different Fissure Sealant Materials and Flowable Composites Used as Pitand-fissure Sealants: A 24-Month Clinical Trial. Paediatr Dent 2015; 37: 468-473.

5. Jafarzadeh M, Malekafzali B, Tadayon N, Fallahi S. Retention of a Flowable Composite Resin in Comparison to a Conventional Resin-Based Sealant: One-year Follow-up. J Dent (Tehran) 2010; 7: 1-5.

6. Cogo E, Calura G. Clinical evaluation of two materials used as pit and fissure sealants: 2 year follow-up. Int J Clin Dent 2009; 2: 241-247.

7. Berger V W. Selection Bias and Covariate Imbalances in Randomized Clinical Trials. John Wiley \& Sons: Chichester, 2005.

8. Berger V W, Exner DV. Detecting selection bias in randomized clinical trials. Control Clin Trials 1999; 20: 319-327.

DOI: $10.1038 /$ sj.bdj.2019.54

The following two letters are in response to a letter 'Tobacco control: Safer without snus' published in the BDJ on 26 October 2018.

Snus

\section{Swedish snus is different}

Sir, recently, a letter by Shanahan raised some concerns about a recent report by the Science and Technology Committee (STC) suggesting the discontinuation of the ban on snus. ${ }^{1}$

However, I believe there are some inaccuracies in the letter that I would like to address. The letter cited a study by Warnakulasuriya ${ }^{2}$ in associating snus with an increased risk for oral cancer. However, that study was mainly referring to oral tobacco products used in Asia, most of which are particularly harmful and associated with elevated risk for oral cancer.

Swedish snus is a very different product. Despite the high rate of snus use among Swedish men, the prevalence of oral cancer in Sweden is among the lowest in the European Union. ${ }^{3}$

There is strong epidemiological evidence that snus use is not associated with a demonstrable increase in oral cancer risk. ${ }^{4,5}$

Therefore, it is particularly important to distinguish Swedish snus from other forms of oral tobacco products which have different toxic potential and substantially elevate the risk for disease.

While correctly mentioned by Shanahan that smoking cessation pharmacotherapies are available and safe, their popularity is limited and they are not used as alternatives by smokers unable or unwilling to quit smoking with the use of approved methods.

For the latter, snus and other tobacco harm-reduction products have a role in substituting for smoking and represent a reasonable option considering that the alternative is to continue to smoke.

Tobacco harm-reduction products do not substitute but supplement other existing tobacco control measures, in an effort to rapidly reduce smoking prevalence and smoking-related disease and death.

Considering that Swedish snus has a long history of strong epidemiological evidence, the suggestion by the STC to review the ban seems reasonable and in fact should be followed by other authorities such as the European Union.

\section{K. Farsalinos, by email}

1. Shanahan D. Tobacco control: Safer without snus. $\mathrm{Br}$ Dent J 2018 225: 685-686.

2. Warnakulasuriya S, Sutherland G, Scully C. Tobacco, oral cancer, and treatment of dependence. Oral Oncol 2005 41: 244-260.

3. La Vecchia C, Lucchini F, Negri E, Levi F. Trends in oral cancer mortality in Europe. Oral Oncol 2004 40: 433439.

4. Luo J, Ye W, Zendehdel K, Adami J, Adami HO, Boffetta $P$, Nyrén O. Oral use of Swedish moist snuff (snus) and risk for cancer of the mouth, lung, and pancreas in male construction workers: a retrospective cohort study. Lancet 2007 369: 2015-2020.

5. Lee P N. Summary of the epidemiological evidence relating snus to health. Regul Toxicol Pharmacol 2011 59: 197-214.

DOI: 10.1038/sj.bdj.2019.55

\section{Much safer with snus}

Sir, I was alarmed to see that poor acquaintance with current oral cancer evidence can lead to backward conclusions as stated in the letter by Dr D. Shanagan.

The referenced study by Foulds et al. was a relevant piece when published 15 years ago, but today several later studies provide much more robust data on the public health benefits that have been reaped from snus use in Sweden. ${ }^{1,2}$

The study by Warnakulasuriya et al. is not only highly outdated but is totally irrelevant with respect to Swedish snus, since it is based on Indian smokeless tobacco products with totally different characteristics. Modern Indian researchers do make the appropriate distinctions resulting in summary statements such as: 'Nasal snuff and snus were not associated with oral cancer risk.'3

The most comprehensive modern summary has been given by the Global Burden of Disease Study 2016 by stating: 'Based on available evidence, for chewing tobacco RRs were significantly higher than one for oral cancer and oesophageal cancer, while for snus or snuff we did not find sufficient evidence of a RR greater than one for any health outcome.'
The suggestions by the Science and Technology Committee (17 August 2018) are actually well supported by the available scientific evidence.

L. M. Ramström, by email

1. Ramström L, Borland R, Wikmans T. Patterns of Smoking and Snus Use in Sweden: Implications for Public Health. Int J Environ Res Public Health. 2016; 13: 1110.

2. Ramström L M, Wikmans T. Mortality attributable to tobacco among men in Sweden and other European countries: An analysis of data in a WHO report. Tob Induc Dis 2014; 12: 14

3. Asthana S, Labani S, Kailash U, Sinha D N, Mehrotra R. Association of Smokeless Tobacco Use and Oral Cancer: A Systematic Global Review and Meta-Analysis. Nicotine Tob Res 2018; DOI: 10.1093/ntr/nty074.

4. GBD 2016 Mortality Collaborators. Global, regional, and national under-5 mortality, adult mortality, age-specific mortality, and life expectancy, 1970-2016: a systematic analysis for the Global Burden of Disease Study 2016. Lancet 2017; 390: 1084-1150.

DOI: $10.1038 /$ sj.bdj.2019.56

\section{Adrenaline use}

\section{The use of pre-filled adrenaline syringes in anaphylaxis kits}

Sir, I am writing with regards to the recent correspondence by the Office of Chief Dental Officer England (OCDO) titled 'Adrenaline for anaphylaxis kits - a reminder to health care professionals.' $^{1}$

This document highlighted the current supply disruption of EpiPen and EpiPen Junior adrenaline auto-injectors (AAIs) and therefore guided dental practitioners and dental care professionals to stock and use adrenaline ampoules in the management of anaphylaxis in order to help preserve national stock of AAIs.

This reminder failed to fully appreciate the use of adrenaline 1:1000 $1 \mathrm{mg} / 1 \mathrm{ml}$ pre-filled syringes when stocking medical emergency kits and their use in the management of anaphylaxis. The use of pre-filled syringes negates the need to stock adrenaline ampoules and to complete the drawing up process.

Reference to pre-filled syringe use is seen in SDCEP guidance and should be more greatly acknowledged as a viable and suitable alternative to stocking adrenaline ampoules in the reminder sent by the OCDO. ${ }^{2}$

Dental professionals are expected to have competency in the use of ampoules and drawing up solutions, however, medical emergencies are rare occurrences, with anaphylaxis accounting for only $1 \%$ of emergencies encountered in the dental setting. ${ }^{3}$

Therefore, the completion of administering adrenaline with the use of ampoules is rarely practiced in real-life emergency situations. We are unaware of any current national 
shortages of adrenaline pre-filled syringes and due to their speed and ease of use relative to ampoules, we feel acknowledgement and advice to stock such equipment should be appreciated in this time of AAI shortage.

A. Travers and K. Taylor, by email

1. Office of Chief Dental Officer England. Adrenaline for anaphylaxis kits - a reminder to Health Care Professionals. NHS Improvement, 2018.

2. SDCEP. Drug Prescribing for Dentistry. Dental Clinical Guidance. Third Edition, 2016.

3. Müller M P, Hänsel M, Stehr S N, et al. A state-wide survey of medical emergency management in dental practices: incidence of emergencies and training experience. Emerg Med J 2008;25: 296-300.

DOI: $10.1038 /$ sj.bdj.2019.57

\section{Medication-related osteonecrosis}

Patients with a hidden risk of MRONJ

Sir, in secondary care we see many cases of medication-related osteonecrosis of the jaw (MRONJ). The referring dentists are often blind-sided by the diagnosis, as their patients may not have disclosed the use any of MRONJ-related medicines. I'd like to highlight to readers a group of patients who should raise suspicion of being at risk of MRONJ.

\section{Signs \& symptoms of osteoporosis ${ }^{2}$}

- Back pain

- Progressive loss of height

- Stooped posture

- Bony fractures

- Kyphosis

- Dowager hump

- Joint/muscle aches

Bisphosphonates (BPs) are the mainstay pharmacological treatment for patients with osteoporosis. Once absorbed, their half-life is over ten years.

They are also used in many other conditions, including metabolic bone disorders and bone cancer. Dentists should have a high index of suspicion in patients with a history of the above conditions, or if they are displaying signs and symptoms of osteoporosis (Box 1).

Discussing their past drug history is important. It is also wise to enquire if they have ever attended hospitals for injectable medications. Intra-venous (IV) BPs place patients at a particularly high risk of MRONJ, and patients often forget about them. ${ }^{3}$

Approximately a fifth of patients on oral BPs will be switched to a different medication due to intolerances. Commonly, these are in the form of gastric irritation or muscoskeletal pain. ${ }^{4}$

Oral agents they can be switched to include vitamin D, calcium, HRT (eg Raloxifene) and strontium ranelate.

Alternatively, they may be switched to parenteral medicines such as IV BPs, parathyroid hormone (teriparatide) or denosumab. ${ }^{5}$

A patient presenting with a combination of any of these medications should be treated with a high index of suspicion for historic $\mathrm{BP}$ use. In such circumstances, we suggest calling the patient's GP practice. With the patient's permission, they can provide a more reliable drug history.

In addition to BPs, other MRONJ-related medicines include RANKL inhibitors (denosumab) and anti-angiogenic drugs (bevacizumab, sunitinib, flibercept). ${ }^{3}$

H. Antov, by email

Drake M T, Clarke B L, Khosla S. Bisphosphonates: mechanism of action and role in clinical practice. Mayo Clinic Proc 2008; 83: 1032-1045.

2. Kanis $J$ A. Diagnosis of osteoporosis and assessment of fracture risk. Lancet 2002:359: 1929-1936.

3. Scottish Dental Clinical Effectiveness Programme. Oral Health Management of Patients at Risk of Medicationrelated Osteonecrosis of the Jaw. Dundee: Dundee Denta Education Centre, 2017.

4. Kennel K A and Drake, M T. Adverse effects of bisphosphonates: implications for osteoporosis management. Mayo Clinic Proc 2009: 84: 632-638.

5. Aspray T J, Francis R M. Treatment of osteoporosis in women intolerant of oral bisphosphonates. Maturitas 2012; 71: 76-78.

DOI: 10.1038/sj.bdj.2019.58 